\title{
Lob für Ärzte, Schelte für Krankenkassen
}

\author{
Seit drei Jahren gibt es das Patientenrechtegesetz - und seit drei Jahren stehen die Normen, die \\ einstiges Richterrecht in Gesetzesform gebracht haben, immer wieder in der Kritik. Den einen geht \\ das Gesetz nicht weit genug, andere fühlen sich gegängelt durch erweiterte Vorgaben wie Doku- \\ mentations- und Aufklärungspflichten. Doch was hat das Gesetz in der Realität gebracht? Dieser \\ Frage ist das IGES Institut im Auftrag des Patientenbeauftragten der Bundesregierung, Karl-Josef \\ Laumann, nachgegangen. Das Ergebnis ist - erwartungsgemäß - durchwachsen.
}

„Viele Menschen kennen das Gesetz und ihre Rechte“, sagte der Patientenbeauftragte Laumann bei der Vorstellung der Studie in Berlin. Für 95 Prozent der Patienten und Versicherten ist das Patientenrechtegesetz nach eigenen Angaben wichtig oder eher wichtig. Dieser hohe Stellenwert geht aus der Studie hervor, für die das IGES Institut nicht nur relevante Literatur, Rechtssprechung und Sekundärdaten ausgewertet hat, sondern auch zahlreiche Interviews mit Patienten, Krankenversicherten, Verbänden, Ärzten und Experten führte.

Neun von zehn Patienten fühlen sich laut der Studie beispielsweise vor einer Operation gut aufgeklärt. Und noch besser: Fast alle Patienten sind mit dem Arzt-Patienten-Gespräch zufrieden. „Solche Werte würden wir nicht erreichen, wenn Ärzte nicht mit ihren Patienten auf Augenhöhe reden würden“, sagte Laumann. Und genau dies sei das erklärte Ziel eines Patientenrechtegesetzes gewesen, das Ungleichgewicht zwischen dem „wissenden“ Arzt und dem auf Hilfe angewiesenen, „unwissenden“ Patienten zu beheben. „Patientenrechte sollten nichts Lästiges sein“, bekräftigte Laumann. „Sie sollten in unserem Gesundheitssystem etwas ganz Normales sein."

\section{Defizite vor allem bei Krankenkassen}

Verbesserungs- und Handlungsbedarf sieht der Patientenbeauftragte allerdings auch noch. Ein Defizit liegt seiner Ansicht nach
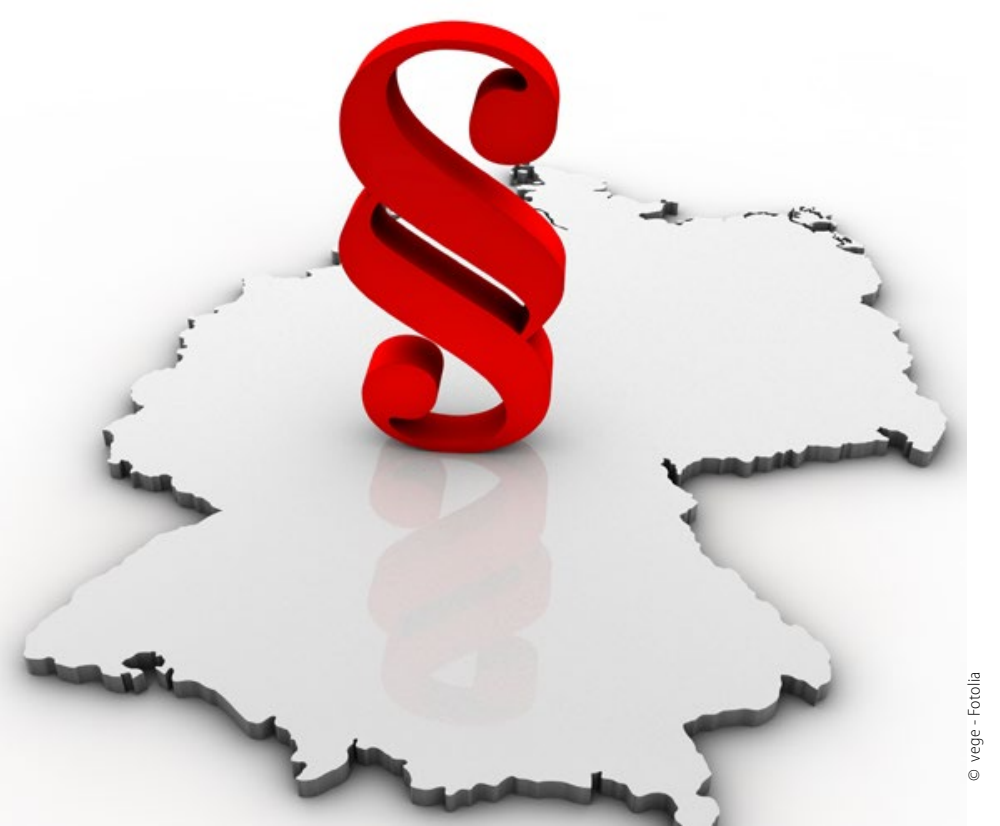

noch immer darin, dass Patienten häufig nicht ohne Komplikationen ihre Krankenakten einsehen könnten, obwohl dies im Gesetz vorgesehen sei. Hier sei mehr Transparenz notwendig. Und auch die Krankenkassen hat Laumann im Visier. Die Studie habe ergeben, dass Krankenkassen in Fragen von eventuellen Behandlungsfehlern den Patienten nicht die Unterstützung zukommen ließen, zu der sie verpflichtet seien. Die Ergebnisse der Studie zeigen, dass etwa 21 Prozent der Befragten angaben, Informationen über Behandlungsfehler nur auf Anfrage zu bekommen. Nur die Hälfte der Befragten weiß zudem, dass die Krankenkassen gesetzlich verpflichtet sind, bei einem Verdacht auf Behandlungsfehler unterstützend tätig zu werden. Das IGES Institut empfiehlt deshalb, kassenübergreifende Standards für die Unterstützung von Patienten bei Behandlungsfehlerverdacht zu etablieren. Darüber hinaus lautet eine weitere Empfehlung, Sanktionierungsmöglichkeiten zu prüfen, wenn Pflichten aus dem Patientenrechtegesetz nicht befolgt würden. Diese sind im Gesetz bisher nicht vorgesehen. Dies sei durchaus ein „Umsetzungshemmnis" für die gesetzlichen Vorgaben.

\section{Finanzielle Ausstattung für Gespräche}

Um eine weitere Verbesserung gerade im Bereich Aufklärung und Beratung durch den Arzt für die Patienten zu erzielen, hat das IGES Institut bereits konkrete Vorschläge erarbeitet. Diese werden allerdings ohne eine Erhöhung der finanziellen Ausstattung für Arzt-Patienten-Gespräche nicht möglich sein. So schlägt das IGES Institut beispielsweise vor, für Ärzte „mehr zeitliche Freiräume für Information und Aufklärung“ zu schaffen und peilt dafür auch eine höhere Vergütung an. Ebenso sollten Krankenkassen auch die Kosten für Dolmetscherleistungen oder gebärdensprachliche Übersetzungen übernehmen. Mit einer Verbesserung des Stellenwerts der „sprechenden Medizin“ rennt das Institut bei Ärzten und Zahnärzten offene Türen ein. $\mathrm{Ob}$ dies in absehbarer Zukunft realistisch ist, bleibt eher fraglich, insbesondere vor dem Hintergrund der stockenden Verhandlungen um eine neue Gebührenordnung für Ärzte (GOÄ).

In einer Podiumsdiskussion konnten die Teilnehmer kein einhelliges Urteil über die Ausgangsfrage „Was hat das Patientenrechtegesetz gebracht" finden. Während Christoph Kranich von der Verbraucherzentrale Hamburg auch drei Jahre nach der Umsetzung des Gesetzes für ein ganz anderes Gesetz, mit deutlich mehr und tiefgreifenderen Rechten für die Patienten, plädierte, fand Dr. Jan Böken von der Bertelsmann Stiftung versöhnliche Worte: „Das Gesetz hat Signalwirkung gehabt“, sagt Böken. „Das Selbstbewusstsein der Patienten, ihre Rechte einzufordern, ist gewachsen."

Sabine Schmit 